\title{
¿ES DIFERENTE EL CICLO ECONÓMICO DE LA REGIÓN DE MAGALLANES RESPECTO DEL CICLO ECONÓMICO NACIONAL?
}

\author{
CRISTIAN M. MONDACA-MARINO ${ }^{a} \&$ JULIO C. ROJAS-MORA ${ }^{b}$
}

\begin{abstract}
RESUMEN
El objetivo de este trabajo ha sido analizar el grado de sincronismo entre el ciclo económico de la Región de Magallanes respecto del ciclo económico nacional para el período 1985-2010. Para determinar el ciclo económico se ha usado el procedimiento de Harding y Pagan (2002) y medido el sincronismo con el índice de contingencia de Pearson. Nuestros resultados muestran evidencia de diferentes grados de sincronismo entre los ciclos económicos regionales respecto del ciclo económico nacional, destacando la región de Magallanes por su menor y mayor volatilidad de su crecimiento económico y por presentar un bajo grado de sincronismo entre su ciclo económico y el ciclo económico nacional, como también con los ciclos de las demás regiones.
\end{abstract}

PALABRAS CLAVE: Ciclo económico regional, puntos de giro, sincronización entre ciclos económicos, procedimiento de Bry y Boschan.

\section{IS DIFFERENT THE REGION OF MAGALLANES BUSINESS CYCLE REGARDING THE NATIONAL BUSINESS CYCLE?}

\begin{abstract}
The objective of this study was to analyze the degree of synchronism between the economic cycle of the Magallanes Region and the national economic cycle for the period 1985-2010. To determine the business cycle has been used the Harding and Pagan (2002) procedure and the synchronization with the Pearson contingency index has been measured. Our results show evidence of different degrees of synchronism between the regional business cycles with respect to the national business cycle, highlighting the region of Magallanes due to its lower and greater volatility of economic growth, and to present a low degree of synchronism between its business cycle and the national business cycle, as well as with the cycles of the other regions.

KEY WORDS: Regional business cycle, Turning point, Synchronization business cycle, Bry and Boschan procedure.

a Instituto de Economía, Universidad Austral de Chile, Los Laureles n³5 Interior, Campus Isla Teja, Valdivia. cristianmondaca@uach.cl

b_Escuela de Ingeniería Civil en Informática, Universidad Católica de Temuco, Rudecindo Ortega 2950, Temuco, Chile. jrojas@inf.uct.cl
\end{abstract}




\section{INTRODUCCION}

En estos últimos años ha surgido una creciente preocupación por estudiar los ciclos económicos (Business cycle) y la ocurrencia de fluctuaciones en la economía, habitualmente llamadas crisis económicas. No es de extrañar esta preocupación, si se considera el gran impacto que tienen las crisis económicas en el tejido productivo y en el mercado laboral, efectos que se han podido ver con claridad en la última gran crisis económica vivida el año 2008, la cual afectó a muchos países del mundo, revitalizando el interés por su estudio a nivel internacional (Ahlborn \& Wortmann, 2017; Mayer et al. 2016).

Sin embargo, los ciclos económicos a nivel regional han tenido menos atención (Artis et al. 2011), a pesar que puede ser de gran interés determinar si las economías regionales responden a cambios en el ciclo nacional, o si existe algún grado de desacoplamiento del ciclo económico nacional. Si las economías regionales presentan una dinámica similar al agregado nacional, esta información ayudaría a estimar el impacto que puede tener una crisis económica en las regiones; o de los efectos que pueden tener políticas macroeconómicas nacionales en el nivel regional.

Por otra parte, si existe un desacople, la utilidad de este tipo de estudios facilitaría el diseño de políticas macroeconómicas de nivel sectorial y regional. Además, ofrecería la oportunidad de identificar grupos de regiones con similitud en sus dinámicas de crecimiento económico, esta característica puede ser útil para optimizar políticas públicas de nivel regional, permitiendo la aplicación de medidas diferenciadas según grupo de regiones.

En este sentido algunos estudios muestran evidencia de que los ciclos económicos regionales pueden ser diferentes de los ciclos económicos a nivel nacional, de estudios realizados en el comportamiento cíclico de los Estados en Estados Unidos y de regiones en países europeos y asiáticos (Artis et al. 2011; Artis et al. 2009; Artis \& Okubo, 2010, 2011).

Que las regiones pueden tener ciclos económicos diferentes al ciclo económico nacional se puede deber a que las regiones tienen un mayor grado de especialización sectorial que el nivel nacional y esto hace que estén más expuestas a shock sectoriales provocados a nivel nacional o del comercio internacional (Artis \& Okubo, 2011). Además pueden existir otros factores como la ubicación, el nivel de industrialización y el capital humano calificado disponible en el mercado laboral y factores no económicos tales como hábitos, patrimonio disponible y la cultura, que pueden condicionar las actividades económicas que se desarrollan en el territorio (Artis et al. 2011; Artis \& Okubo, 2010).

La principal aportación de este artículo es mostrar antecedentes del grado de sincronismo entre el ciclo económico de la Región de Magallanes respecto del ciclo económico nacional. Utilizando la metodología de Harding y Pagan (2002) para caracterizar el ciclo económico de las regiones chilenas, y midiendo el grado de sincronismo con la propuesta de Artis et al. (1997).

Cobra especial interés estudiar el caso de Chile, debido al liderazgo de la economía chilena en el contexto latinoamericano, destacando en las últimas décadas por su crecimiento económico sostenido y su estabilidad macroeconómica y experimentado muy pocos episodios de recesión económica. Sin embargo, existen estudios que mencionan la disparidad en el crecimiento económico de las regiones y la heterogeneidad en términos de tamaño, participación, capacidad productiva y mercado laboral (Aroca \& Bosch, 2000; Aroca \& Hewings, 2002; Atienza \& Aroca, 2012).

Además, el factor territorial parece tener un efecto claramente diferenciador de las actividades económicas que se desarrollan en las regiones de Chile, debido a la distancia, clima, estacionalidad, y características geográficas propias y diferentes entre regiones, con lo cual, se puede suponer un comportamiento heterogéneo en las dinámicas de crecimiento económico; y se podría plantear la hipótesis de la existencia de diferencias en el comportamiento de los ciclos económicos regionales respecto del ciclo económico nacional y diferencias y similitudes entre regiones.

En este contexto regional heterogéneo, destaca la Región de Magallanes por su condición de zona extrema, situación que ha permitido disfrutar de ciertas ventajas económicas, como poseer una zona franca con impuestos reducidos especiales, ó el disfrute de asignaciones especiales 
para los sueldos de los empleados públicos por parte del Estado. Estas ventajas han condicionado en cierta manera la dinámica de su economía y de su mercado laboral (Ferrada \& Montaña, 2014).

Además su economía está basada en la explotación de recursos naturales (Ramírez \& Lira, 2008; Soza-Amigo \& Aroca, 2012), con un bajo impacto en las remuneraciones para los habitantes de la región (Aroca \& Soza-Amigo, 2013), rasgo que puede condicionar el comportamiento de su economía, producto de crisis sectoriales y contracción de la demanda interna/externa de los mercados vinculados a sus principales actividades económicas; ó las dinámicas de contratación/ expansión de sectores con ciclos extractivos y temporales. Por otra parte, el Estado participa en forma importante en su economía (vía transferencias y subsidios) a través de sus empresas públicas y de organismos del Estado, que generan una alta demanda de bienes y servicios en la región.

Por todo lo mencionado anteriormente, la elección de la región de Magallanes se justifica en la medida que por sus características particulares de lejanía geográfica, su especificidad de actividades económicas, diferente funcionamiento de su mercado laboral y baja conectividad geográfica con otras regiones (se conecta sólo con la región de Aysén en su extremo norte), posibilitaría una dinámica de crecimiento económico diferente al del agregado nacional.

El documento tiene la siguiente estructura; primero introduce el tema y su importancia, en la sección dos se presenta una breve revisión sobre el estudio de los ciclos económicos a nivel general y en el estudio de los ciclos regionales. En la sección tres se presenta la metodología y los datos utilizados en el estudio; en la sección cuatro se presentan los resultados, y finalmente, en la sección cinco se presentan las conclusiones.

\section{EL ESTUDIO DE LOS CICLOS ECONÓMICOS}

Los ciclos económicos son fluctuaciones recurrentes, pero no periódicas, que afectan a la economía en su conjunto cambiando su dinámica de crecimiento. Está constituido de a lo menos dos fases de comportamiento ${ }^{1}$; una fase de expansión de las actividades económicas, seguido por una fase de decaimiento o contracción de las actividades, hasta que nuevamente comienza un período de expansión de las actividades económicas (Burns \& Mitchell, 1946).

Una buena parte del esfuerzo de investigación se ha centrado en desarrollar metodologías para detectar los momentos en los cuales la economía cambia su régimen normal de crecimiento y experimenta fluctuaciones; es decir, establecer con certeza en qué momento se ha alcanzado un máximo relativo (peak) en el nivel de la actividad económica y cuándo ha ocurrido su valor mínimo relativo de actividad (trough); ambos momentos son conocidos como puntos de giro (turning points), y con ellos la posibilidad de establecer una cronología, comúnmente llamada fechado de las fases del ciclo (Moore \& Zarnowitz, 1984).

La más clásica de las metodologías utilizadas para estudiar el ciclo económico fue desarrollada por el The National Bureau of Economic Research (NBER), entidad radicada en Estados Unidos, que monitorea este tipo de fenómenos desde principios del siglo veinte. En sus comienzos, la metodología consideraba la observación de cientos de series económicas para determinar los momentos en los cuales ocurrían cambio en el comportamiento de crecimiento de la economía en su conjunto. En la actualidad y, debido al conocimiento del fenómeno, se ha reducido significativamente el número de variables a ser observadas, centrando la atención en aquellas variables macroeconómicas que tienen un comportamiento coincidente con el ciclo económico, como son el producto interior bruto (PIB) o el índice de producción industrial (IPI).

Un aspecto relevante de la metodología del NBER es incluir la opinión de expertos que utilizan diversos criterios cuantitativos y cualitativos para detectar los puntos de giro. Por este motivo, en el transcurso del tiempo se han desarrollado otras metodologías que evitan el recurrir a la valoración de expertos y concentran su esfuerzo en desarrollar herramientas cuantitativas para la caracterización del ciclo económico, definidas con criterios objetivos y que sean replicables (Boldin, 1994).

De las metodologías propuestas, destaca la presentada por Bry y Boschman (1971), que

1 Algunos autores caracterizan más fases, ver por ejemplo Harding y Pagan (2002). 
desarrollan un procedimiento para determinar puntos de giro, utilizando reglas simples de decisión a través de las cuales se seleccionan puntos de giro potenciales, que luego se contrastan de acuerdo con un número de restricciones previamente acordadas. Este procedimiento busca proporcionar un método objetivo y fácilmente replicable, que sea capaz de entregar resultados similares a los entregados por el NBER, siendo utilizado en diversos estudios debido a su simpleza (Harding \& Pagan, 2002; King \& Plosser, 1994; McDermott \& Scott, 2000; Mejías-Reyes et al. 2005; Watson, 1994).

Se debe comentar que en la literatura existe a veces una confusión respecto del objeto de estudio, confundiendo habitualmente el estudio del ciclo económico clásico (business cycle) con el ciclo de desviaciones a la tendencia (growth cycle). El primero, analiza las series de niveles, coincidentes con el comportamiento del ciclo económico, fenómeno no observable directamente, $y$ en donde lo central es determinar la ocurrencia de los períodos de recesión de la economía. En el segundo, se analizan las desviaciones del producto respecto de su valor de tendencia en el largo plazo, usualmente obtenida por un método de filtrado (por ej. Hodrick \& Prescott, 1997), con el cual se obtiene una componente cíclica que caracterizan los períodos de crecimiento y decrecimiento de la economía respecto de la tendencia, además de las fluctuaciones provocadas por recesiones ocurridas en la economía; a pesar que en muchos artículos no se aclare la diferencia.

Como se mencionó anteriormente, la mayoría de los trabajos que han estudiado el ciclo económico clásico, se han enfocado en el análisis a nivel nacional y su comparación a nivel internacional, determinando los grados de similitud entre ciclos de países, bloques comerciales, o regiones integradas monetariamente, como la zona Euro (Mejia-Reyes, 1999, 2002). O la determinación de ciclos comunes que afecten a múltiples economías a la vez (Mondaca, 2012, p. 37).

Sin embargo, el estudio de los ciclos económicos regionales no ha recibido la misma atención en las investigaciones, y son escasos y recientes los estudios que analizan los ciclos económicos clásicos en una escala regional, de los cuales se puede citar los siguientes trabajos: Artis et al. (2011); Artis y Okubo (2010); Artis y Okuboy (2011); Di Gresia y Garegnani (1999) y Gadea et al. (2006).

Sorprende este panorama, si se considera la gran utilidad que puede tener este tipo de estudios para ayudar a generar políticas públicas adecuadas al contexto económico regional. Por ejemplo, si las economías regionales presentaran dinámicas de crecimiento y fluctuación diferentes a las del nivel nacional, políticas públicas uniformes y homogéneas causarian efectos heterogéneos a nivel regional y serían convenientes políticas contextualizadas a la realidad de cada región o grupo de regiones a fines.

Además, ocasionaría ineficiencia en el uso de los recursos públicos, debido a que no se generarían las medidas adecuadas que cada región o grupo de regiones necesitan para gestionar de mejor forma el comportamiento de su crecimiento regional y, en particular, revertir crisis económicas de nivel regional, a pesar que el nivel nacional no esté experimentando una recesión.

Este escenario de diferentes comportamientos entre los ciclos económicos regionales y el nacional, parece posible debido a que fluctuaciones en el crecimiento económico regional pueden ser causados no sólo por el ciclo económico nacional, sino también por otros factores. Por ejemplo, algunas regiones tienden a ser más sensibles a shock particulares (del comercio internacional o ajustes sectoriales) debido a que tienen un grado mayor de especialización productiva que el de nivel nacional (Artis et al. 2011).

También -si se pudiera conocer el impacto del ciclo económico nacional en las economías regionales- se podría implementar medidas más efectivas en incentivar el crecimiento económico y el empleo a nivel regional. Además se podría evaluar el efecto de las políticas sectoriales/macroeconómicas nacionales en el crecimiento económico regional ó evaluar el grado de exposición a las crisis internacionales/nacionales de las economías regionales, entre otros interesantes temas.

En el caso de Chile, los estudios de los ciclos económicos regionales son aún más escasos, existiendo sólo un trabajo que aborda la problemática del sincronismo entre ciclos de desviaciones a la 
tendencia de las regiones chilenas y ninguno que analice los ciclos económicos clásicos regionales, es decir, desde la perspectiva clásica.

En este caso, Sarrias y Aroca (2012) estudian el sincronismo entre ciclos de crecimiento, es decir, las desviaciones del crecimiento respecto de una hipotética tendencia, para las regiones de Chile en el período 1988-2005. Para obtener la componente cíclica aplican el filtro de Hodrick y Prescott (1997), para luego modelar el grado de sincronismo con algunos indicadores macroeconómicos como la inversión pública y privada. En este caso, sus resultados mencionan el bajo grado de sincronismo entre el ciclo nacional y el ciclo de las regiones.

\section{METODOLOGÍA}

Para el estudio del ciclo económico de las regiones se ha utilizado el producto interno bruto (PIB) anual regionalizado para el período 1985-2010², que considera la división territorial vigente hasta el año 2007, es decir, 13 regiones individualizadas por números romanos como sigue: región de Tarapacá (I), región de Antofagasta (II), región de Atacama (III), región de Coquimbo (IV), región de Valparaíso (V), región de O’Higgins (VI), región del Maule (VII), región del Biobío (VIII), región de la Araucanía (IX), región de Los Lagos (X), región de Aysén (XI), región de Magallanes (XII) y región Metropolitana de Santiago de Chile (R.M.).

Las series del PIB anuales han sido trimestralizadas para mejorar la resolución del procedimiento de fechado, utilizando el método de Denton-Cholette (Dagum \& Cholette, 2006, p. 80). Para determinar los puntos de giro se ha utilizado el algoritmo de (Bry \& Boschan, 1971) en su versión para datos trimestrales propuesta por Harding y Pagan (2002).

Este procedimiento utiliza requisitos de amplitud y consta de cuatro etapas:

1. Se identifican y reemplazan los valores extremos ${ }^{3}$ por la media de los valores adyacentes.

2. Se identifican los puntos de giro

Disponible en www.bcentral.cl

3 Se definen los valores extremos como aquellos que presentan una tasa de cambio obtenida como diferencias de logaritmos, respecto de los trimestres adyacentes de distinto provisionales en una serie suavizada por medias móviles ${ }^{4}$, garantizándose su alternancia:

a. Identificación de los puntos más altos (más bajos) en un entorno de cuatro trimestres a cada lado.

b. Cumplimiento de la alternancia de puntos de giro, seleccionando el más alto de los puntos máximos múltiples (el más bajo de los puntos mínimos múltiples).

3. Se identifican los puntos de giro provisionales en la serie original (sin alisar) exigiéndose una amplitud mínima a las fases de al menos un error estándar de los cambios trimestrales en logaritmo y una duración de los ciclos mínima de cinco trimestres (peak-peak o trough-trough).

a. Identificación de los puntos más altos (más bajos) en un entorno de cuatro trimestres a cada lado.

b. Cumplimiento de la alternancia de puntos de giro, seleccionando el más alto de los peak múltiples (el más bajo de los trough múltiples).

c. Identificación de los segmentos planos.

d. Identificación y exclusión de los valores extremos entre los posibles puntos de giro.

e. Cumplimiento de la alternancia de puntos de giro, seleccionando el más alto de los peak múltiples (el más bajo de los trough múltiples).

f. Identificación de ciclos cortos (menores de cinco trimestres).

g. Aplicación de la regla de amplitud mínima que exige un error estándar (de los cambios en logaritmos) de separación entre el peak y el trough del ciclo identificado.

4. Determinación de aquellos puntos de giro que presentan correspondencia en los dos conjuntos de puntos de giro obtenidos en la segunda y tercera etapa, con una tolerancia de \pm 1 trimestre y eliminación de los demás puntos no coincidentes.

Este procedimiento ha sido utilizado por

signo y superior a 3 errores estándares de la serie de tasas de crecimiento $\ln \left(\mathrm{y}_{\mathrm{t}}\right)-\ln \left(\mathrm{y}_{\mathrm{t}-1}\right)$.

4 Los máximos y mínimos de la serie en la media móvil de \pm 2 trimestres. 
Mejia-Reyes (2003) y Mondaca-Marino, (2012, p. 86) en trabajos de determinación del ciclo económico de países a nivel latinoamericano.

Para la determinación del grado de sincronismo entre ciclos económicos regionales se ha utilizado la metodología propuesta por Artis et al. (1997) que utilizan el coeficiente de correlación muestral y de contingencia de Pearson corregido, para determinar el grado de similitud entre fluctuaciones de dos ciclos. Este indicador sólo considera la coincidencia entre la ocurrencia de períodos de recesión y expansión de la economía, ignorando sus amplitudes (Mondaca 2012, p. 92).

El método utiliza una variable binaria creada por la cronología de los ciclos económicos clásicos, asociando ceros a los años de expansión y unos a los de contracción, para cada uno de los ciclos considerados. Luego para medir la similitud entre dos ciclos, se compara dos series binarias y se obtiene una tabla de contingencia de orden $2 \mathrm{x} 2 \mathrm{y}$ con ésta se estima el coeficiente de contingencia de Pearson corregido ${ }^{5}$ :

con

$$
\begin{gathered}
C C_{\text {corr }}=\sqrt{\frac{\chi^{2}}{N+\chi^{2}}} \cdot \frac{100}{\sqrt{0.5}} \\
\chi^{2}=\sum_{i=0}^{1} \sum_{j=0}^{1} \frac{\left[n_{i j}-n_{i} n_{j} / N\right]^{2}}{n_{i} n_{j} / N}
\end{gathered}
$$

Donde $n_{i j}(i, j)=\{0,1\}$, representa el número de períodos en los que dos regiones (o una región y el país) están en alguna de las siguientes situaciones: en expansión ambos, en recesión ambos, en recesión y expansión; y en expansión $y$ recesión. En este caso $\mathrm{N}$ es el número total de observaciones.

Si las dos variables binarias que definen los regímenes del ciclo son independientes 6 entonces $n_{i j}=n_{i} n_{j}, y C_{\text {corr }}$ por tanto es igual a cero. En el otro extremo, con dependencia absoluta, el coeficiente es igual a 100; si bien esta situación incluye relaciones

5 Se denomina corregido debido a que está relacionado con el coeficiente de contingencia convencional que en el caso de una tabla de contingencia de dimensión finita, el valor máximo obtenible está determinado por la dimensión de la tabla. En concreto, el valor máximo obtenible para este coeficiente en nuestro caso es $\sqrt{0.5}$, término que se utiliza para la corrección.

6 En nuestro caso la independencia implica que no hay procíclicas y contra cíclicas. Este estadístico ha sido utilizado para estudiar el sincronismo entre ciclos económicos por Artis et al. (1997), Mejía-Reyes (2003, p. 70) y Mondaca (2012, p. 92) entre otros autores. A continuación se presentan los resultados obtenidos en este trabajo.

\section{RESULTADOS}

En la Fig. 1 y Tabla 1, se puede observar el comportamiento de las tasas de crecimiento del PIB regional para el período 1985-2010. Chile experimentó un crecimiento anual promedio del $4,4 \%$, valor por sobre la media de crecimiento que experimentó América Latina y el Caribe (LAC), que para el mismo período fue de sólo un 3,0\%.

Este importante nivel de crecimiento experimentado a nivel nacional en el período 1985-2010 oculta las diferentes dinámicas de crecimiento económico que se pueden observar a nivel regional (ver Fig. 1), donde existen regiones con crecimientos superiores al promedio del país (como son las regiones I, II, III, VII, IX, X y XI) y regiones que presentan un crecimiento promedio inferior a la media nacional (como son las regiones $\mathrm{V}$, VI, VIII y XII). Este panorama es aún más heterogéneo si se considera las importantes variaciones en el crecimiento que han sufrido las regiones chilenas (ver Tabla 1), con regiones que presentan altos valores de crecimiento en el período (ejemplo de ellos son las regiones II y III) y otras regiones en las cuales su crecimiento ha sido muy estable en el tiempo (ejemplo de ello son las regiones $\mathrm{V}$, VI, VIII y $\mathrm{X}$ ).

Además se puede observar un comportamiento muy volátil, existiendo momentos en los cuales el crecimiento del PIB ha estado por sobre el $10 \%$, destacando las regiones II y III, con crecimientos de un $22,8 \%$ y $18,4 \%$ respectivamente. También se puede observar regiones que en algunos momentos han experimentado fuertes caídas en su crecimiento, como son las regiones III, XI y XII,

correlación contemporánea entre los regímenes del ciclo económico para los casos analizados. En el caso de dependencia completa, indica que los dos regiones (o una región y el país) están en el mismo régimen en cada periodo de tiempo, o bien, siempre en el opuesto y que por lo tanto tiene idénticos puntos de giro, pudiendo ser opuesto su tipo. 


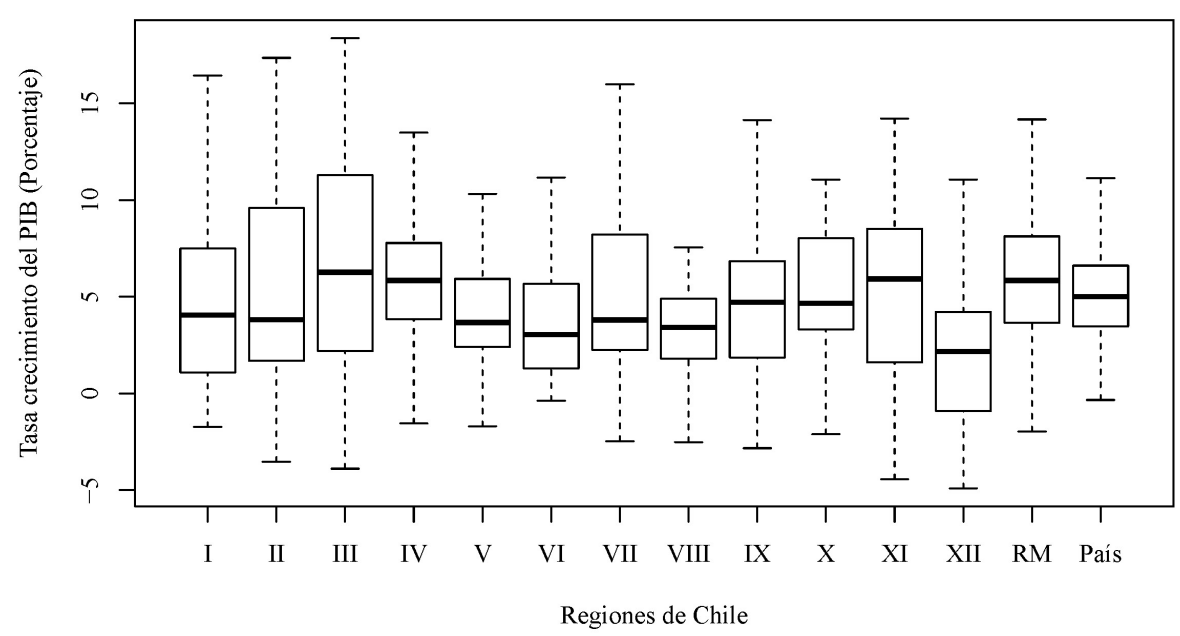

Fig. 1. Diagrama de caja y bigote de las tasas de crecimiento del PIB regional y del país (1985-2010).

Tabla 1. Tasas de crecimiento promedio, máxima, mínima y desviación estándar del PIB regionalizado período 1985-2010.

\begin{tabular}{lccccccccccccc}
\hline \multicolumn{1}{c}{ Regiones } & I & II & III & IV & V & VI & VII & VIII & IX & X & XI & XII & País \\
\hline Promedio & $5,2 \%$ & $5,5 \%$ & $6,8 \%$ & $5,7 \%$ & $4,2 \%$ & $4,0 \%$ & $5,0 \%$ & $3,3 \%$ & $4,9 \%$ & $5,3 \%$ & $5,3 \%$ & $1,4 \%$ & $4,4 \%$ \\
\hline Max & $16,4 \%$ & $22,8 \%$ & $18,4 \%$ & $20,2 \%$ & $10,3 \%$ & $11,2 \%$ & $16,0 \%$ & $7,6 \%$ & $14,1 \%$ & $11,1 \%$ & $14,2 \%$ & $11,1 \%$ & $8,6 \%$ \\
\hline Min & $1,7 \%$ & $3,5 \%$ & $-3,9 \%$ & $-3,7 \%$ & $-1,7 \%$ & $-0,4 \%$ & $-2,5 \%$ & $-2,5 \%$ & $-2,8 \%$ & $-2,1 \%$ & $-4,4 \%$ & $-9,3 \%$ & $-1,6 \%$ \\
\hline D.E. & 0,05 & 0,07 & 0,06 & 0,05 & 0,03 & 0,03 & 0,04 & 0,03 & 0,04 & 0,03 & 0,05 & 0,04 & 0,02 \\
\hline
\end{tabular}

con tasa negativas de un $-3,9 \%,-4,4 \%$ y $-9,3 \%$ respectivamente.

En este aspecto, la región de Magallanes (XII) destaca por presentar un crecimiento promedio inferior al de las demás regiones del país, producto de una mayor volatilidad en su crecimiento, que presenta valores altos de crecimiento, pero también valores de crecimiento negativos muy altos, mayor al de otras regiones. Estas características del crecimiento de Magallanes puede ser evidencia de una mayor vulnerabilidad de la economía en escenarios de crisis económica nacional o en sectores relevantes de su economía, y una menor capacidad de crecimiento en los períodos de bonanza económica.

Un aporte de esta investigación es la propuesta de un fechado del ciclo económico para cada una de las regiones del país, que se puede observar en la Tabla 2, que además incluye un resumen estadístico que caracteriza los rasgos característicos de los ciclos económicos regionales de Chile.
Nuestros resultados muestran que el ciclo económico de las regiones de Chile se caracteriza por una duración promedio de 24,6 trimestres $(6,2$ años), con una fase de contracción de 3,7 trimestres (0,9 años) de duración y una fase de expansión de 21,2 trimestres (5,3 años) de duración. Además en promedio, las regiones ha experimentado 3,6 períodos de recesión en sus economías. Diferente del comportamiento a nivel nacional en donde se puede ver el ciclo económico que se caracteriza por una duración de 39,0 trimestres $(9,75$ años), con sólo tres períodos de contracción ó recesiones económicas y una duración de su fase de contracción de 3,5 trimestres ( 0,8 años) y de 36,0 trimestres ( 9 años) su fase de expansión, que demuestra la diferencia entre el comportamiento del ciclo económico nacional respecto de los ciclos económicos a nivel regional.

Analizando el comportamiento de los ciclos económicos regionales se puede apreciar que son diferentes en la recurrencia de las contracciones $y$ en los períodos de expansión. Por ejemplo las 


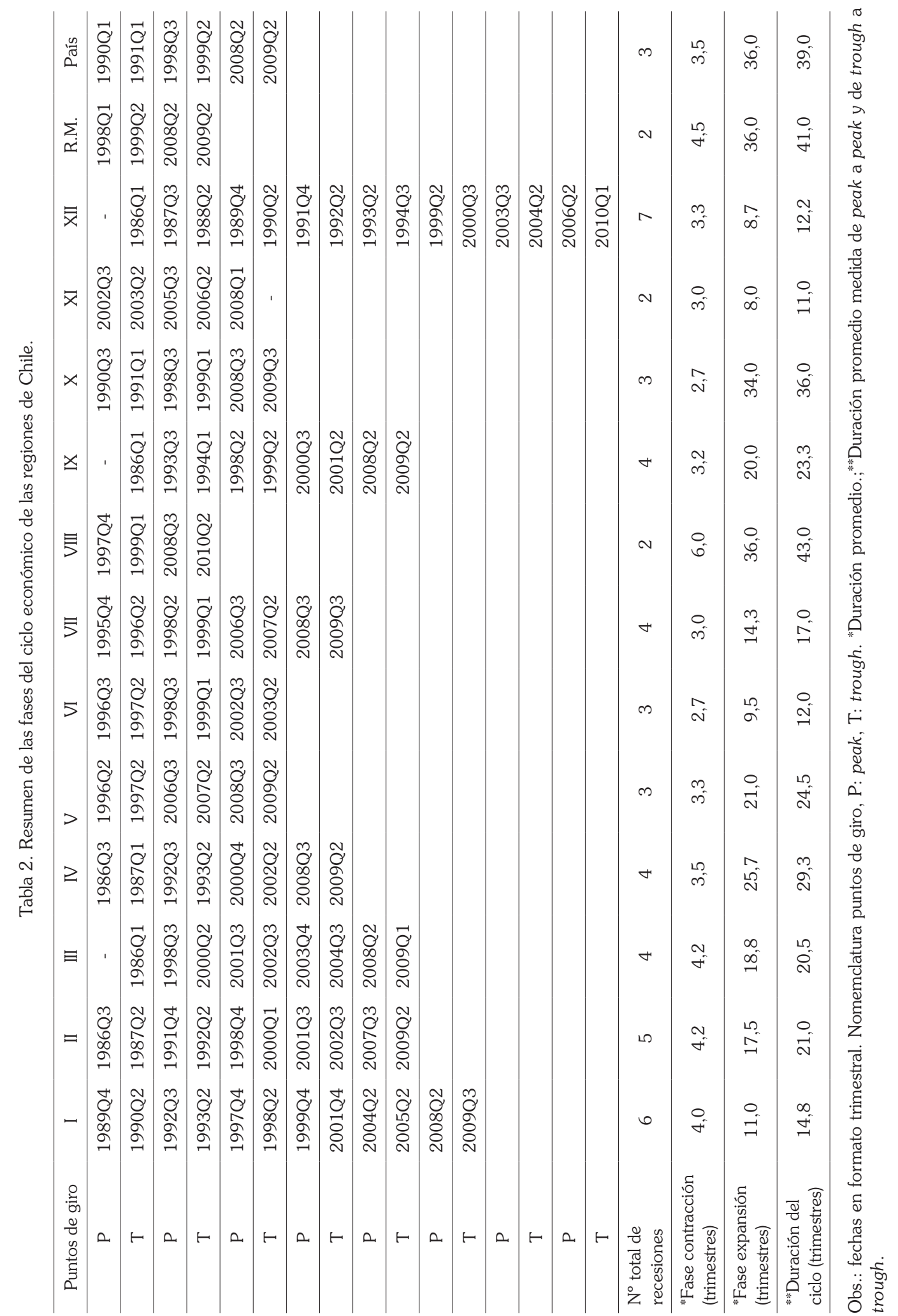


regiones XI y R.M. presentan sólo dos contracciones en período de análisis, versus, las regiones I y XII que presentan seis y siete contracciones en sus ciclos económicos respectivamente. Comentario similar se puede hacer con respecto a las duración promedio de las fases de contracción, con períodos muy breves como los característicos de la región $\mathrm{X}$ que duran en promedio 2,7 trimestres y otros muy extensos como los que caracterizan al ciclo de la región VIII con una duración media de seis trimestres. En el caso de las fases de expansión se observan diferencias significativas, con períodos muy breves como los ocurridos en las regiones XI y XII que duran en promedio ocho y 8,7 trimestres respectivamente; y otras regiones con períodos muy extensos como las regiones VIII y R.M. que duran en promedio 36 trimestres ambos.

A pesar de las diferencias que se observan entre los ciclos regionales entre si, y entre ellos y el ciclo nacional, también se puede observar diferencias en el comportamiento del ciclo, con una fase recesión de mayor duración que el observado a nivel nacional y con períodos de expansión más breves que a nivel nacional. Estos antecedentes muestra la mayor vulnerabilidad de la economía regional a los shocks económicos de nivel nacional que se sobreponen a las dinámicas internas de la región.

A nivel individual destacan las regiones VIII, X y R.M. por un menor número de períodos recesivos y una menor duración en el tiempo de los mismos, y con fases expansivas de más larga duración que las fases de las demás regiones. En este sentido, se puede decir que son regiones que se han caracterizado por un crecimientos económico estable en el tiempo, aunque con diferentes ritmos de crecimiento (ver Fig. 1 y Tabla 1).

También destacan las regiones I (Tarapacá) y XII (Magallanes) por tener la mayor cantidad de períodos de recesión ó crisis económicas; y aun cuando la duración de los períodos de recesión no son de los más largos observables a nivel regional, esta situación condiciona la duración de las fases expansivas ó de crecimiento sostenido que experimentan estas regiones, los cuales como consecuencia, son de menor duración que el de las demás regiones.

Estos rasgos evidencia una inestabilidad y fragilidad del crecimiento económico sostenido que presentan estas regiones, situación que se puede deber a una mayor exposición frente a los choques externos de diverso origen (crisis internacionales, nacionales, o sectoriales) respecto de las demás regiones y una menor capacidad de crecimiento sostenido debido a su condición de zonas extremas.

En la Fig. 2 se presenta el ciclo económico de la región de Magallanes (a) y el ciclo económico del país (b), en donde se pueden observar que el ciclo económico de Magallanes presenta un número mayor de períodos de contracción o recesión que el ciclo económico nacional. En este caso, siete contracciones, algunas de ellas muy intensas en algunos de los períodos, particularmente en el último de ellos.

A nivel nacional en cambio, el ciclo económico se caracteriza por sólo tres períodos a)

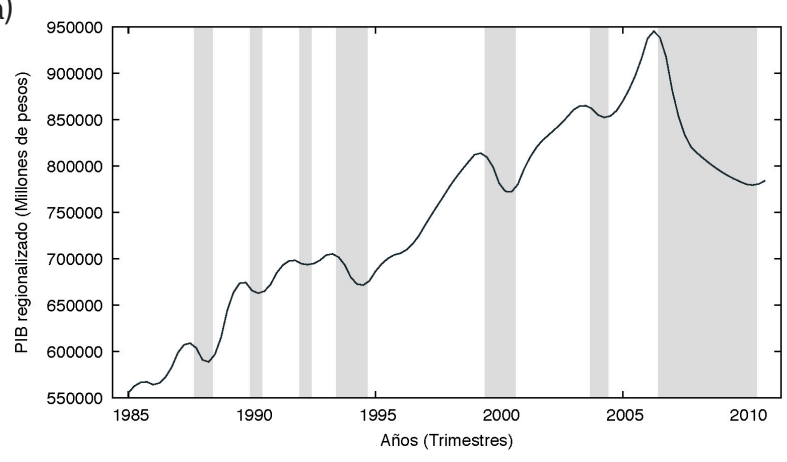

b)

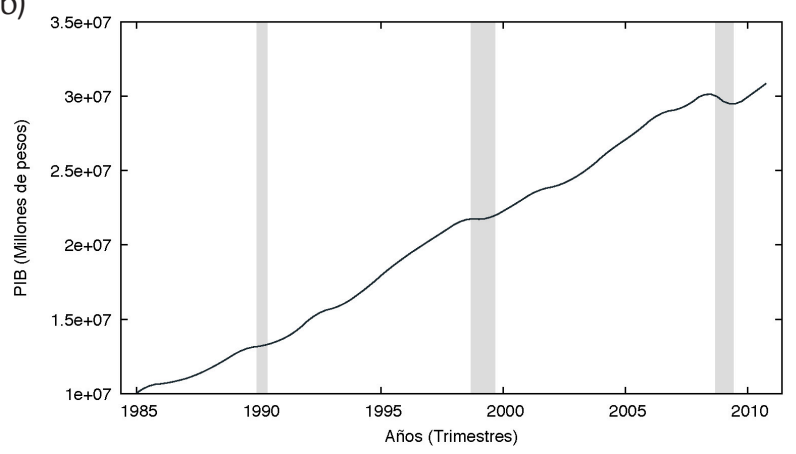

Fig. 2. PIB regionalizado de Magallanes (a) y de Chile (b) (período 1985-2010).

*Áreas en gris períodos de recesión identificados del ciclo económico en cada caso. 
de recesión y con fluctuaciones menos intensas que las observables en el ciclo de Magallanes. Los períodos de contracción a nivel nacional están asociados a crisis económicas de nivel internacional que afectaron a la economía chilena como fueron "la crisis del peso mexicano" en el año 1990, "la crisis Asiática" en el año 1998, y "la crisis de las hipotecas Subprime" que afecta en forma rezagada a Chile desde mediados del 2008 hasta mediados del 2009 (Mondaca, 2012, p. 305).

Es interesante destacar que de ambos ciclos se puede identificar sólo un único período de contracción de nivel nacional que coincide con una contracción en el ciclo económico de la región de Magallanes y que ocurre el año 2009. En este caso, se puede observar que Magallanes ya presentaba una dinámica de contracción desde antes de la crisis de nivel internacional que se solapa con la ocurrida el 2009, lo que probablemente provocó un retraso en la recuperación del crecimiento sostenido en la economía regional, la cual se recupera recién el 2010.

Para complementar este análisis hemos medido el grado de similitud entre ciclos económicos regionales, y entre estos y el de nivel nacional, utilizando el índice de contingencia de Pearson. En la Tabla 3 se puede observar un resumen de los valores de sincronismo obtenidos en donde se puede destacar que existen pares de regiones con un alto nivel de sincronismo o similitud entre sus ciclos económicos, es decir, sus ciclos económicos presentan rasgos similares entre sí, particularmente en los períodos de expansión de la economía, como son las regiones: R.M., X, VIII y VII. En el otro extremo, se puede observar un grupo de regiones con un bajo nivel de sincronismo, como son las regiones XII, XI, I y VI. En este aspecto, destaca nuevamente la región de Magallanes que en promedio presenta el más bajo valor de sincronismo entre ciclos económicos con las demás regiones $(17,6)$.

Al considerar el grado de sincronismo de los ciclos económicos regionales respecto del ciclo económico nacional, se puede apreciar nuevamente un grupo de regiones con un alto valor del indicador, como son las regiones VIII, IX, $\mathrm{X}$ y R.M, es decir, estas regiones presentan rasgos comunes al ciclo nacional, particularmente en los períodos de expansión de la economía nacional.

También se puede observar regiones con un bajo nivel de sincronismo con el ciclo económico nacional como son las regiones I, IV,VI y XII. En este caso, destaca el bajo grado de sincronismo entre el ciclo económico de Magallanes y el ciclo

Tabla 3. Sincronismo entre los ciclos económicos de las regiones y del país (1985-2010).

\begin{tabular}{|c|c|c|c|c|c|c|c|c|c|c|c|c|c|c|}
\hline & I & II & III & IV & V & VI & VII & VIII & IX & $\mathrm{x}$ & XI & XII & $\mathrm{R}, \mathrm{M}$, & País \\
\hline I & - & 10,4 & 23,9 & 56,7 & 2,3 & 30,3 & 11,5 & 33,8 & 26,4 & 17,7 & 1,8 & 3,5 & 36,1 & 25,2 \\
\hline II & & - & 56,5 & 50,4 & 7,1 & 2,6 & 17,3 & 22,7 & 19,0 & 32,3 & 15,8 & 15,6 & 50,1 & 58,5 \\
\hline III & & & - & 20,5 & 2,4 & 12,7 & 17,3 & 22,7 & 53,4 & 32,3 & 0,0 & 27,7 & 50,1 & 58,5 \\
\hline IV & & & & - & 18,9 & 22,0 & 12,2 & 16,5 & 27,6 & 24,1 & 4,9 & 25,8 & 24,1 & 30,5 \\
\hline $\mathrm{V}$ & & & & & - & 34,1 & 69,5 & 26,6 & 12,5 & 34,1 & 15,6 & 14,3 & 34,1 & 40,3 \\
\hline $\mathrm{VI}$ & & & & & & - & 15,9 & 19,5 & 7,3 & 26,0 & 20,9 & 44,4 & 26,0 & 31,4 \\
\hline VII & & & & & & & - & 66,7 & 40,8 & 67,8 & 18,2 & 9,8 & 67,8 & 66,4 \\
\hline VIII & & & & & & & & - & 46,4 & 72,5 & 50,2 & 10,1 & 80,2 & 71,0 \\
\hline IX & & & & & & & & & - & 47,6 & 6,5 & 28,9 & 71,6 & 72,2 \\
\hline $\mathrm{X}$ & & & & & & & & & & - & 31,5 & 2,6 & 72,2 & 78,9 \\
\hline $\mathrm{XI}$ & & & & & & & & & & & - & 23,1 & 31,5 & 38,6 \\
\hline XII & & & & & & & & & & & & - & 6,3 & 16,1 \\
\hline R.M. & & & & & & & & & & & & & - & 94,3 \\
\hline País & 25,2 & 58,5 & 58,5 & 30,5 & 40,3 & 31,4 & 66,4 & 71,0 & 72,2 & 78,9 & 38,6 & 16,1 & 94,3 & - \\
\hline Promedio & 21,5 & 27,6 & 29,1 & 25,7 & 24,0 & 22,5 & 37,0 & 41,5 & 35,4 & 41,5 & 19,9 & 17,6 & 49,6 & 52,5 \\
\hline
\end{tabular}


económico del país $(16,1)$. Este resultado muestra evidencia que el ciclo económico de Magallanes es independiente del ciclo económico nacional y que predominan comportamientos endógenos, y que probablemente la economía regional no disfruta de los efectos positivos del crecimiento económico nacional, o incluso acentuar los períodos de recesión internos de las regiones.

Llama la atención que de nuestros análisis previos, las regiones de Tarapacá (I) y de Magallanes (XII) destacan por sus marcadas diferencias respecto del comportamiento de las demás regiones y respecto del comportamiento del ciclo económico nacional. Resultados similares a los presentados por Ramírez y Lira (2008) y Aroca y Soza-Amigo (2013).

Los diferentes grados de sincronismo existente entre las regiones y el agregado nacional presenta dos interesantes resultados. Para el caso de alto grado de sincronismo, es probable que estas regiones disfruten en mayor medida de los períodos de expansión de la economía nacional, o en sentido inverso, la economía nacional se puede ver beneficiada con el crecimiento de éstas economías regionales; pero también es probable que éstas regiones sufran, en forma más intensa y en forma rápida, la desaceleración del crecimiento económico, y los períodos de recesión, con lo cual, si coinciden con períodos de recesión endógenos, propios de la región, pueden ocurrir procesos de retroalimentación e intensificación de las crisis económicas de nivel nacional en las regiones.

Debido a esto puede ser aconsejable disponer de políticas de crecimiento económico que consideren estas diferencias y concentrar los esfuerzos de inversión pública en aquellas regiones que estén más sincronizadas con el ciclo económico nacional para revertir rápidamente períodos de crisis económicas, en vez de aplicar políticas estandarizadas y uniformes a todas las regiones en forma simultanea, que beneficiaría a aquellas regiones que no se ven afectadas en forma inmediata de una crisis económica de nivel nacional, pero no permitiría focalizar recursos en aquellas regiones que más lo necesitan.

En el caso de las regiones con un bajo nivel de sincronismo con el ciclo económico nacional, se puede concluir que las dinámicas cíclicas de estas regiones son independientes de la coyuntura económica nacional, y en donde probablemente predominan comportamientos endógenos, muy diferentes de la dinámica de crecimiento nacional. En este contexto, las economías regionales probablemente no disfrutan de los efectos positivos del crecimiento económico nacional, e incluso, podrían sufrir con los períodos de recesión del ciclo económico nacional, agravando sus períodos de recesión endógenos o aminorando los resultados de sus períodos de expansión.

Por lo tanto, sería muy recomendable la creación de políticas económicas que no sólo se activen en los períodos de crisis, sino que intervengan en los períodos de bonanza económica nacional, para redistribuir recursos en aquellas regiones con dificultades en su crecimiento económico.

Respecto de la región de Magallanes, se pudo determinar que es una región con una dinámica en su crecimiento económico particular muy diferente al del país y al de las demás regiones, caracterizado por un crecimiento económico volátil e inestable. Estos rasgos muestran una menor capacidad de la economía de Magallanes para recuperarse en los períodos de recesión, o para aprovechar los períodos de expansión de la economía nacional. Además el grado de sincronismo de su ciclo económico respecto del ciclo nacional es bajo, debido a que su ciclo se caracteriza por una mayor ocurrencia de períodos de recesión, una mayor duración de los mismos y períodos de expansión más breves.

Estos antecedentes nos permite responder a la pregunta inicial, respecto de la diferencia o similitud del ciclo económico de Magallanes y el ciclo económico nacional, en donde es evidente que Magallanes presenta una dinámica cíclica diferente que el observado en el ciclo económico nacional, reflejando esto, las profundas diferencias en cuanto al funcionamiento de las economías regionales en general y diferencias en las dinámicas de crecimiento económico que los caracterizan.

Estos resultados son similares a los obtenidos por Aroca y Bosch (2000) respecto de que existen comportamientos de crecimiento diferentes entre regiones $y$ que existe un componente geográfico que puede condicionar 
el crecimiento de las regiones. Según nuestros resultados con datos más actualizados, esta situación no parece cambiar. Además la existencia de grupos de regiones con rasgos similares de crecimiento también es un resultado que se confirma de forma similar a los hallazgos de Ramírez y Lira (2008).

\section{CONCLUSIONES}

En esta investigación se ha determinado el grado de sincronismo entre el ciclo económico clásico de la Región de Magallanes respecto del ciclo económico clásico Nacional, proponiendo una novedosa cronología de los ciclos económicos para las regiones de Chile para el período 19852010. Para evaluar el sincronismo entre ciclos económicos se utilizó el coeficiente de contingencia de Pearson aplicado a las fases cíclicas obtenidas por la metodología propuesta por Harding y Pagan (2002).

De los principales resultados se pudo detectar un comportamiento muy diferente entre el comportamiento de los ciclos económicos regionales y el ciclo económico nacional medidos por el grado de sincronismo. Esto debido probablemente a las diferencias dinámicas de crecimiento existentes entre regiones.

Además se observan más períodos de recesión, con una mayor duración y períodos de expansión más breves, que los existentes a nivel nacional. Estos rasgos evidencia una inestabilidad en el crecimiento económico regional, y por lo tanto, las regiones chilenas en general parecen estar más expuestas a las fluctuaciones de sus economías que a nivel nacional, particularmente las regiones extremas de Chile, como son la región de Magallanes y la región de Tarapacá.

Un comportamiento cíclico regional diferente al observado a nivel nacional pueden ser ocasionado por las diferentes participaciones en el agregado nacional que presentan las regiones, ó por sus diferencias en las relaciones comerciales que muestran las regiones entre sí, antecedentes mencionados por Atienza y Aroca (2012), pero pueden existir otros factores como la dinámica de crecimiento sectorial, la disponibilidad del factor trabajo, o la productividad existente en los territorios, aspectos que sugieren un análisis desde la perspectiva de una función de producción agregada de nivel regional.

En el caso de la región de Magallanes, se pudo determinar que su crecimiento económico presenta una dinámica muy diferente al del nivel nacional, caracterizado por una mayor volatilidad y fluctuaciones más intensas en su crecimiento, una mayor ocurrencia y duración de sus períodos de recesión y una menor duración de sus períodos de expansión, respecto de lo que se observa a nivel nacional. Por todos estos antecedentes, se puede afirmar que la región de Magallanes sí presenta un ciclo económico diferente al nacional.

Respecto de líneas futuras de investigación, dado los resultados obtenidos en este trabajo, es evidente que las dinámicas de crecimiento económico a nivel regional son diferentes $y$, por tanto, sería interesante investigar los factores que pueden estar causando estas diferencias, particularmente analizando el comportamiento cíclico del crecimiento sectorial en las regiones. Aroca y Soza-Amigo (2013) entregan antecedentes preliminares sobre diferencias existentes entre las estructuras productivas de dos regiones (Magallanes y Arica) respecto de la estructura productiva a nivel nacional.

Por otra parte, las diferentes dinámicas de crecimiento regional pueden estar condicionadas por la cantidad y calidad de trabajo disponible, y el efecto concentrador que pueden tener algunas regiones en capturar el trabajo disponible. Antecedentes al respecto son presentados por Aroca y Hewings (2002) sobre la mayor probabilidad de la Región Metropolitana (R.M.) en captar migrantes de otras regiones, que las demás regiones. Sin embargo, se debería complementar este análisis respecto de la calidad (capital humano acumulado) que disponen las regiones para producir sus bienes y servicios. Abordar la problemática de la productividad de los factores productivos a nivel regional y su dinámica puede también ayudarnos a explicar el por qué de las diferentes dinámicas de crecimiento de las economías regionales, facilitando el diseño de políticas públicas de crecimiento de nivel regional, que aborden las diferentes facetas del crecimiento económico que se observan en las regiones chilenas. 


\section{AGRADECIMIENTOS}

Los autores desean agradecer los comentarios de los revisores y sus sugerencias. Este trabajo ha sido financiado por la Dirección de Investigación y Desarrollo de la Universidad Austral de Chile (proyecto S-2014-08, El ciclo del empleo y el ciclo económico en Chile).

\section{BIBLIOGRAFÍA}

Ahlborn, M., \& Wortmann, M. (2017). The core-periphery pattern of European business cycles: A fuzzy clustering approach. Journal of Macroeconomics, 55, 12-27.

Aroca, P., \& Bosch, M. (2000). Crecimiento, convergencia y espacio en las regiones chilenas: 1960 - 1998. Estudios de Economia, 27(2), 199-224.

Aroca, P., \& Hewings, G. (2002). Migration and regional labor market adjustment: Chile 1977-1982 and 1987-1992. Annals of Regional Science, 36, 197-218.

Aroca, P., \& Soza-Amigo, S. (2013). Diferencias productivas estructurales entre el Centro y la Periferia: Magallanes y Arica versus el promedio nacional. Magallania, 41(2), 101-118.

Artis, M., Dreger, C., \& Kholodilinf, K. (2009). Common and spatial drivers in regional business cycles, (SERCDP0022).

Artis, M., Dreger, C., \& Kholodilin, K. (2011). What drives regional business cycles? The role of common and spatial components. Manchester School, 79(5), 10351044.

Artis, M., Kontolemis, Z., \& Osborn, D. (1997). Business Cycles for G7 and European Countries. The Journal of Business, 70(2), 249-279.

Artis, M., \& Okubo, T. (2010). The UK intranational business cycle. Journal of Forecasting, 29(1-2), 71-93.

Artis, M., \& Okubo, T. (2011). The intranational business cycle in Japan. Oxford Economic Papers, 63(1), 111-133.

Atienza, M., \& Aroca, P. (2012). Concentración y crecimiento en Chile: una relación negativa ignorada. EURE (Santiago), 38(114), 257-277.

Boldin, M. (1994). Dating turning points in the business cycle. Journal of Business, 67(1), 97-131.

Bry, G., \& Boschan, C. (1971). Cyclical Analysis of Time Series: Selected Procedures and Computer Programs. NBER Technical Paper (Vol. 20).

Burns, A., \& Mitchell, W. (1946). Measuring business cycles. NBER Books (Vol. I).

Dagum, E., \& Cholette, P. (2006). Benchmarking, temporal distribution, and reconciliation methods for time series. Springer.

Di Gresia, L., \& Garegnani, M. (1999). Relación entre ciclo económico regional y nacional: análisis del período 1961-1995. Económica, XLV(4), 261-281.

Ferrada, L., \& Montaña, V. (2014). ¿Existe una Ventaja de Salario para los Habitantes de la Región de Magallanes?: Análisis a Partir de un Enfoque por Género. Magallania (Punta Arenas), 42(1), 93-109.

Gadea, M., Goméz, A., \& Montañes, A. (2006). How many regional business cycles are there in Spain ?: a MS VAR approach (Documento de Trabajo No. 27/2006).

Harding, D., \& Pagan, A. (2002). Dissecting the cycle: A methodological investigation. Journal of Monetary Economics, 49(2), 365-381.

Hodrick, R. J., \& Prescott, E. C. (1997). Postwar U.S. Business Cycles: An Empirical Investigation. Journal of Money, Credit and Banking, 29(1),1-16.

King, R., \& Plosser, C. (1994). Real business cycles and the test of the Adelmans. Journal of Monetary Economics, 33(2), 405-438.

Mayer, E., Rüth, S., \& Scharler, J. (2016). Total factor productivity and the propagation of shocks: Empirical evidence and implications for the business cycle. Journal of Macroeconomics, 50, 335-346.

McDermott, C. J., \& Scott, A. (2000). Concordance in business cycles (IMF Working Paper No. 37).

Mejia-Reyes, P. (1999). Classical business cycles in Latin America: turning points, asymmetries and international synchronisation. Estudios Económicos.

Mejia-Reyes, P. (2002). Why national business cycles are largely independent in Latin America? Evidence from intraregional trade and investment. Ciencia Ergo Sum, 9(1), 10-20.

Mejia-Reyes, P. (2003). No-linealidades y ciclos económicos en América Latina. (El Colegio Mexiquense, Ed.). D.F. Mexico: Universidad Autónoma del Estado de México.

Mejías-Reyes, P., Martinez, J., \& Rendón, W. (2005). Investigación económica. Investigación Económica, 92(254), 91-124.

Mondaca, C. (2012). Estudio de sincronismos entre ciclos económicos mediante la transformada wavelet: análisis del caso Chile y Mercosur. Universidad de Valladolid.

Moore, G., \& Zarnowitz, V. (1984). The development and role of the National Bureau's business cycle chronologies (NBER Working Paper No. 1394).

Ramirez, J., \& Lira, I. (2008). Globalization and regional development: The economic performance of Chile's 
regions, 1990-2002. Cepal Review, 95, 103-123.

Sarrias, M., \& Aroca, P. (2012). Regional Business Cycle Synchronization in Chile (Documento de Trabajo).

Soza-Amigo, S., \& Aroca, P. (2012). Análisis y simulaciones en base a las matrices insumo-producto de la comuna de
Punta Arenas para los años 2003 y 2007. Magallania (Punta Arenas), 40(2), 113-127.

Watson, M. (1994). Business-cycle durations and postwar stabilization of the US economy. American Economic Review, 84(1), 24-46. 